\title{
COMMENTARY
}

\section{Community members co-designing a trial of medication access}

\author{
Nav Persaud MD MSc, Marleane Davidson, Diane Charter
}

Cite as: CMAJ 2018;190(Suppl 1):S44-S45. doi: 10.1503/cmaj.180415

CMAJ Podcasts: author interview at https://soundcloud.com/cmajpodcasts/patient-oriented-research

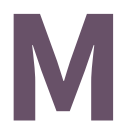

any Canadian patients face barriers to medication access that negatively affect their health. ${ }^{1}$ Medications are still excluded from the publicly funded health care system in Canada, despite multiple government and independent recommendations, as well as international standards. ${ }^{2}$ Clear demonstrations that publicly funding medications will save billions of dollars have not led to policy change. ${ }^{3}$ We designed the CLEAN Meds (Carefully Selected and Easily Accessible at No Charge Medications) study to help bridge an evidence-policy gap around medication access by measuring the effects of providing people with free and convenient access to medications, while incorporating community members as partners. The research team engaged with those who are directly or indirectly affected by barriers to medication access to generate findings that are pertinent to the concerns of patients and to increase the likelihood that the research would help inform public policy. ${ }^{4-6}$

Co-designed by a community guidance panel, the CLEAN Meds study (ClinicalTrials.gov NCT02744963) is an open (not blinded), parallel two-arm, superiority, individually randomized controlled trial conducted in three primary care sites in Ontario, Canada. ${ }^{7}$ Adult patients ( $\geq 18 \mathrm{yr}$ ) who reported nonadherence to medications because of cost were eligible to participate in the study. Participants were randomly assigned to two groups: one to receive free and convenient access to a carefully selected list of essential medications $(n=395)$ and the second to receive the usual means of medication access $(n=391)$. Care for patients in both groups was otherwise unchanged. The primary outcome of this trial was adherence to appropriately prescribed medications. ${ }^{7}$

The research team aimed to engage community members as partners and to involve them in many aspects of the study to make this research patient-oriented. We used a variety of techniques for finding people who were interested in co-designing this study and would reflect the diversity of the population affected by poor access to medications. The most successful approach was to canvass the neighbourhoods surrounding St. Michael's Hospital in downtown Toronto (one of the proposed study sites). Members of the research team and several medical students wore T-shirts reading "Free medicines?" and distributed a postcard inviting community members to join the community guidance panel. We also contacted potential members through existing community groups and through random digit dialing. ${ }^{4}$

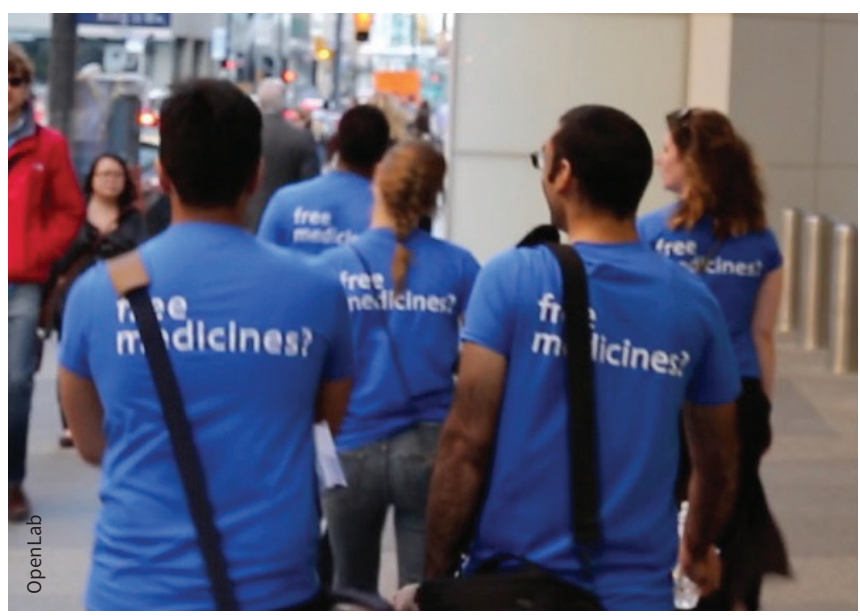

Members of the research team canvas downtown Toronto for community guidance panel members.

\section{KEY POINTS}

- In an effort to bridge the policy gap around medication access, we engaged patient partners in the design of a randomized controlled trial, the CLEAN Meds (Carefully Selected and Easily Accessible at No Charge Medications) study.

- A community guidance panel co-designed the trial and helped ensure the research was patient-oriented.

- Follow-up with the community guidance panel on actions taken based on the panel's input keeps the research team accountable and demonstrates the value of the decisions made by the panel.

Through these methods, we established a community guidance panel of 12 members that meets face-to-face every one to two months. At the outset, key decision-makers, such as the assistant deputy minister and executive officer of the Ontario Public Drug Programs, were invited to these meetings to discuss how research can inform public policy with the community guidance panel. The panel members co-designed many aspects of the study, including designing the intervention and selecting the trial outcomes; some other design decisions were made by the research team and reviewed by the panel. 
Important decisions were made during meetings through open discussion among members of the research team and panel members. For example, the protocol for the pharmacist contacting participants after dispensing medications through the mail was discussed in detail with the panel; a decision was made that the pharmacist should call participants one week after dispensing medications. The panel established a process for determining which participants would be eligible for a home visit from the pharmacist. The panel also decided on several trial outcomes. In addition to outcomes directly related to medications, the panel decided to include an outcome related to whether participants were better able to make ends meet (since providing free medications could make it easier for people to manage financially without changing medication use).

We involved panel members in crucial aspects of the study conduct, including the data-collection plan, for which they helped to design scripts for contacting participants and suggested what time of day may be convenient for participants to complete the survey over the telephone. They also participated in selection committees when candidates were interviewed as we hired new research staff members.

The community guidance panel co-designed the knowledge translation plan that includes traditional outlets, such as journal publications and conference presentations, and also innovative plans such as a documentary film exploring the experiences of trial participants. Patient engagement will continue with further knowledge translation planning and execution, and patients will be involved in designing future studies.

We used the Public and Patient Engagement Evaluation Tool to assess the process of engaging people through our panel. ${ }^{8}$ Panel members and members of the research team completed surveys after six months, and we collectively discussed the findings. We also regularly invited feedback from members of the panel.

We adjusted several aspects of the engagement process, including the method of compensation. We stopped providing the type of food that is often offered at meetings in hospitals and instead provided healthy food. The most important lesson learned from earlier experience was the need to report back to the panel on the follow-up work the research team did based on the panel's input at the previous meeting. We added this to the agenda of every meeting, as it keeps the research team account- able and demonstrates the value of the decisions made by the panel. We now make certain study decisions during the meeting. Before ending the discussion of an agenda item, we go around the table to ensure that everyone has an opportunity to express a view and come to a final decision on the topic.

Direct benefits to the community guidance panel members included compensation for their time attending meetings, reimbursement for travel and other expenses, teaching about research methods (as requested by members of the community guidance panel) and a sense of ownership over the project. We invited researchers to discuss aspects of the research process, such as the rationale for random allocation of participants. Some community guidance panel members participated in capacitybuilding events hosted by OSSU (the Ontario SPOR [Strategy for Patient-Oriented Research] SUPPORT [Support for People and Patient-Oriented Research and Trials] Unit).

We hope that the work of the panel will help increase the likelihood that the findings from the trial will inform public policy changes, which could in turn benefit panel members and the Canadian population at large.

\section{References}

1. Prescription drug access and affordability an issue for nearly a quarter of all Canadian households. Vancouver: Angus Reid Institute; 2015 July 15. Available: http://angusreid.org/wp-content/uploads/2015/07/2015.07.09-Pharma.pdf (accessed 2018 Oct. 3).

2. Morgan SG, Daw JR. Canadian pharmacare: looking back, looking forward. Healthc Policy 2012;8:14-23.

3. Federal cost of a national pharmacare program. Ottawa: Office of the Parliamentary Budget Officer; 2017 Sept. 28.

4. Domecq JP, Prutsky G, Elraiyah T, et al. Patient engagement in research: a systematic review. BMC Health Serv Res 2014;14:89.

5. Manafo E, Petermann L, Mason-Lai $P$, et al. Patient engagement in Canada: a scoping review of the 'how' and 'what' of patient engagement in health research. Health Res Policy Syst 2018;16:5.

6. Oxman AD, Lewin S, Lavis JN, et al. SUPPORT Tools for evidence-informed health Policymaking (STP) 15: engaging the public in evidence-informed policymaking. Health Res Policy Syst 2009;7(Suppl 1):S15.

7. Persaud N, Lee T, Ahmad H, et al. Protocol for a randomised controlled trial evaluating the effects of providing essential medicines at no charge: the Carefully seLected and Easily Accessible at No Charge Medicines (CLEAN Meds) trial. BMJ Open 2017;7:e015686.

8. Abelson J, Li K, Wilson G, et al. Supporting quality public and patient engagement in health system organizations: development and usability testing of the Public and Patient Engagement Evaluation Tool. Health Expect 2016;19:817-27
More information on this project is available at www.ossu.ca/IMPACTAwards.

Competing interests: Nav Persaud reports grants from the Canadian Institutes of Health Research (CIHR) and OSSU (the Ontario SPOR [Strategy for Patient-Oriented Research] SUPPORT [Support for People and Patient-Oriented Research and Trials] Unit). No other competing interests were declared.

This article was solicited and has been peer reviewed.
Affiliations: Department of Family and Community Medicine (Persaud), University of Toronto; St. Michael's Hospital (Persaud); Centre for Urban Health Solutions (Persaud, Davidson, Charter), Keenan Research Centre, Toronto, Ont.

Contributors: Nav Persaud drafted the manuscript. All of the authors contributed to the conception of the work, revised the content and gave approval of the version to be published. All of the authors are accountable for all aspects and integrity of the work.
Funding: The CLEAN Meds study is funded by OSSU and CIHR.

Acknowledgements: The authors acknowledge the contributions of all members of the community guidance panel.

Disclaimer: Nav Persaud is an associate editor for CMAJ and was not involved in the editorial decision-making process for this article.

Correspondence to: Nav Persaud, Nav. persaud@utoronto.ca 